\title{
INTEGRAL and Small Telescopes
}

\author{
Nami Mowlavi, Peter Kretschmar, Marc Türler, Nicolas Produit \\ INTEGRAL Science Data Center \\ 16 ch. d'Ecogia, CH-1290 Versoix, Switzerland
}

\begin{abstract}
The future INTEGRAL satellite, to be launched in 2002, will observe the universe in gamma-rays. Parallel observations in other wavelengths are desirable for many gamma-ray objects, the most famous being certainly the gamma-ray bursts.

In this article, we present the INTEGRAL mission and its scientific objectives. We then give three examples of astrophysics fields for which combined gamma ray and optical observations are recommended. Small telescopes would be ideally suited for many of the studies.
\end{abstract}

\section{Introduction}

If human eyes were sensitive to gamma-ray photons $(\mathrm{E} \geq 100 \mathrm{keV})$, an astronaut in space would see a much more variable sky than what we see in the optical. The high energy universe is populated with objects whose luminosities vary on a wide time scale from milliseconds to years. The most famous of these objects are certainly the gamma ray bursts (GRBs), due to the mystery which veiled their very nature since their discovery in the fifties up to recent years. A better understanding of these objects was only made possible when the optical counterpart of a GRB was first observed simultaneously with the GRB event (van Paradijs et al. 1997). This stresses the importance of simultaneous multiwavelength observations, particularly for high energy astrophysics where the duration of an event can be less than a day. The role of small $(<1 \mathrm{~m})$ class telescopes is essential in this respect.

The next big gamma-ray mission is the INTErnational Gamma-Ray Astrophysics Laboratory (INTEGRAL). It is planned to be launched in 2002 by the European Space Agency. Its nominal life mission is two years, extendible to five years. The wide interest of the astrophysical community in this mission is attested by the large number of proposals submitted during the first announcement of opportunity (due on February 2001). The total requested observing time for the first year of the mission exceeded the available open time by a factor of 19 .

In this contribution, we emphasize the role that small telescopes can play during INTEGRAL's mission. Section 2 describes the instruments on board of INTEGRAL and Sect. 3 the scientific objectives of the mission. Three examples of high energy astrophysics fields of study of potential interest for small telescopes are presented in Sect. 4. Conclusions are drawn in Sect. 5. 


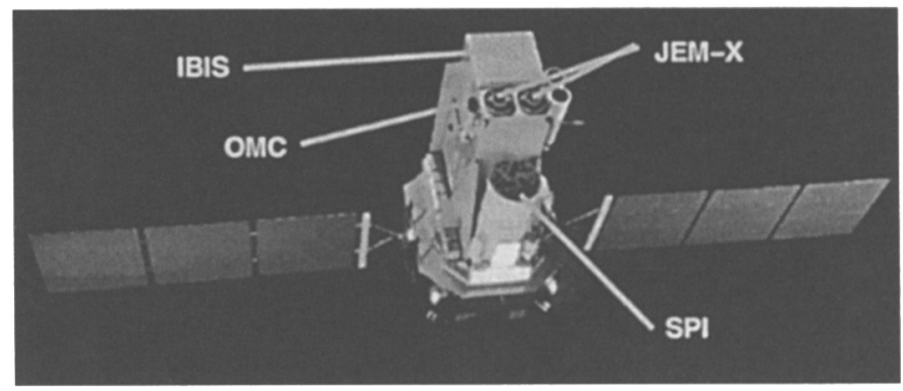

Figure 1. INTEGRAL with its instruments.

\section{INTEGRAL and its Instruments}

The INTEGRAL satellite is shown in Fig. 1. It comprises two main $\gamma$-ray instruments, a spectrometer (SPI) and an imager (IBIS), and two secondary instruments, an X-ray telescope (JEM-X, which is actually duplicated) and an optical monitoring camera (OMC).

The SPI spectrometer is sensitive from $20 \mathrm{keV}$ to $8 \mathrm{MeV}$. It is composed of 19 cooled, hexagonally shaped, high purity Ge detectors, designed to achieve an optimal spectral resolution of $3 \mathrm{keV}$. Its field of view covers 16 degrees with an angular resolution of 2 degrees.

The IBIS imager is designed to achieve an optimal spatial resolution of 12 arcmin. It is made of thousands of small, fully independent pixels, and operates in the energy range $20 \mathrm{keV}$ to $10 \mathrm{MeV}$.

The two identical X-ray imagers are built to extend the $\gamma$-ray observations down to energies of $3 \mathrm{keV}$. The angular resolution is 1 arcmin.

Finally, the optical monitoring camera enables to observe stars up to a magnitude of 19.7. It has a field of view of 5 degrees and a resolution of 17.6 arcsec.

All three high energy telescopes (SPI, IBIS and JEM-X) are coded mask instruments to localize the direction of a source. This technique is required because of the difficulty to focalize $\gamma$-ray photons. The coded mask apertures of SPI is clearly visible in Fig. 1 (mosaic on top of the instrument).

During the mission, the data from the satellite is received in near real time at the INTEGRAL Science Data Center (ISDC) located in Geneva, Switzerland, where it is analyzed for fast detection of GRBs and transients. Consolidated data is later processed and archived, and private data distributed to the observers in the scientific community. Further information can be found at http://isdc.unige.ch.

\section{Science with INTEGRAL}

INTEGRAL deals with energies from about $1 \mathrm{keV}$ to $10 \mathrm{MeV}$. This corresponds to temperatures $T$ from $10^{7}$ to $10^{11} \mathrm{~K}$. The physical conditions which are able to produce such energetic photons can be identified from a rough estimation of the kinetic, gravitational and nuclear energies. These are considered in turn 
in the following paragraphs. The actual physical processes which convert these energies into $\gamma$-ray photons are mentioned at the end of this section.

The kinetic energy of a particle of rest mass $m_{0}$ can be estimated by equating the thermal energy $\frac{3}{2} k T$ ( $k$ is the Boltzmann constant) with the relativistic kinetic energy $(\gamma-1) m_{0} c^{2}$ ( $\gamma$ being the Lorentz factor $1 / \sqrt{1-v^{2} / c^{2}}$, with $c$ the speed of light). This shows that $v / c \simeq 0.1$ for protons at $10 \mathrm{MeV}$, while electrons are relativistic at energies above $1 \mathrm{MeV}$. INTEGRAL will thus deal with relativistic electrons.

Let us now consider the energy acquired by a particle in a potential well around an object of mass $M$ and radius $R$. Equating the potential energy $G m_{0} M / R$ ( $G$ being the gravitational constant) to $3 / 2 k T$ gives the relation $M / R=3.1 \times 10^{-9} \times T / m_{0}$, masses, radius and $T$ being expressed in $\mathrm{g}, \mathrm{cm}$ and $\mathrm{K}$, respectively. To reach an energy of $10 \mathrm{MeV}\left(\sim 10^{11} \mathrm{~K}\right)$, a proton must thus be in the potential well of an object with $M / R \simeq 1.8 \times 10^{26} \mathrm{~g} / \mathrm{cm}\left(\simeq 3.4 \times 10^{29} \mathrm{~g} / \mathrm{cm}\right.$ for electrons). White dwarfs have $M / R \simeq 4 \times 10^{23} \mathrm{~g} / \mathrm{cm}$, and are thus not dense enough to meet the requirement. Neutron stars, with $M / R \simeq 2 \times 10^{26} \mathrm{~g} / \mathrm{cm}$, are good candidates to accelerate protons to the required energies. The $\gamma$ ray astrophysical fields of interest to INTEGRAL will thus include accretion phenomenon around neutron stars or black holes. We note that clusters of galaxies also provide a deep potential well able to accelerate particles to $\gamma$-ray energies.

Transitions between two nuclear states of a given nucleus also provide a source for $\gamma$-ray emission. An example is the $1.8 \mathrm{MeV}$ line from ${ }^{26} \mathrm{Al}$ disintegration.

For highly magnetic neutron stars $\left(B \gtrsim 10^{1} 1\right.$ Gauss), the spectra are also influenced by the quantization of energies at these field strength (Landau levels) leading to observable line features.

Emission processes at the origin of $\gamma$-ray photons include:

- black body radiation (surface of neutron stars);

- bremsstrahlung (electrons accelerated in the Coulomb field of atomic nuclei);

- synchrotron radiation (relativistic electrons in a magnetic field);

- Compton processes (electron-photon diffusion) with either thermal or relativistic electrons;

- relativistic jets that boost either process;

- nuclear line emission (also from annihilation processes).

\section{Examples of High Energy Astrophysical Objects}

\subsection{Active Galactic Nuclei}

Active galactic nuclei (AGN) are very compact and luminous sources located at the very center of galaxies. The most distant and luminous AGNs are called quasars (contraction of quasi-star because they appear in the visible as faint stars of $\mathrm{m}_{\mathrm{V}}>12.5$ ). They emit about $10^{48} \mathrm{erg} / \mathrm{s}$ and are thus the most powerful persistent sources in the universe.

Some quasars have the peculiarity to emit over the whole electromagnetic spectrum, from the radio to the gamma-ray domain (Fig. 2). This very broad spectrum can only be explained by many different emission processes arising at 


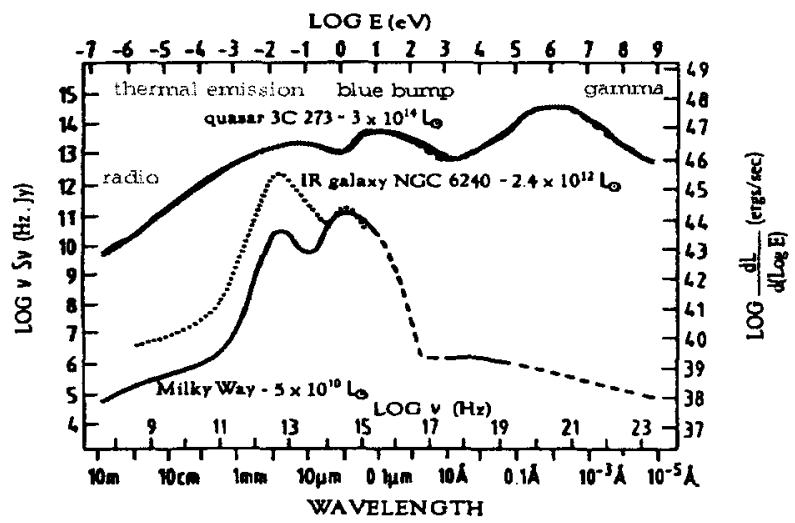

Figure 2. Comparison of the spectral energy distribution of a typical quasar (3C 273), an infrared galaxy (NGC 6240) and a normal galaxy (the Milky Way). Adapted from Camenzind (1997).

different places around a central super-massive black hole (e.g. Robson 1996). In current models, the radiation from the near-infrared to the ultra-violet would be due to thermal emission from an accretion disk and a dusty torus surrounding the back hole. The radio component is most probably synchrotron emission arising in the jets observed to stream out from the central source. Hard X-ray and gamma-ray radiations are thought to be inverse-Compton emission due to thermal or synchrotron photons scattered by the relativistic electrons in the jet.

The luminosity of the so-called blazars, a subtype of quasars, varies by a factor of two or more on a time scale between a few days to a few months. Although variability is observed at all wavelengths, the amplitude and the variability pattern can be very different from one spectral band to another (Türler et al. 1999). This differential variability helps to distinguish the various emission components of the source. The study of multi-wavelength variability thus has the potential to put strong constraints on the various emission processes at work in AGN. For the bright quasar 3C 273, for instance, this approach has already proven to be successful for the study of the blue-bump (Paltani et al. 1998), the millimeter-to-radio emission from the jet (Türler et al. 2000) and the relationship between the infrared and the X-ray emission (McHardy et al. 1999).

The continuous monitoring of AGN at all possible wavelengths is the key towards a better understanding of the physics at work in these still poorly known objects. In this respect, small telescopes are ideally suited to monitor AGNs in the optical domain simultaneously to the gamma-ray observations performed by INTEGRAL.

\subsection{X-Ray Binaries}

X-ray binaries are composed of a compact object - usually a black hole or a neutron star - which accretes mass from its binary companion. The binary companion is in most cases still on or close to the main sequence. There are three basic accretion mechanisms. The first one is the Roche-lobe overflow from 
the companion when the latter has evolved enough to increase its radius beyond the Roche lobe. It is the only mechanism which allows mass transfer from lowmass companions. The second accretion mechanism is wind accretion from the strong stellar wind of a massive star, and the last one is accretion from the equatorial outflow disk of a Be star.

The enormous gravitational potential energy released by mass accretion on these compact objects ( $>0.1 m c^{2}$ compared to $0.007 m c^{2}$ for nuclear burning of hydrogen) powers the production of high energy radiation. For black holes, the observed radiation must be produced in the accreted matter while it is drawn in, which is usually performed through an accretion disk. The dramatic spectral changes that are observed for many black hole candidates can be explained by changes in the properties of the accretion flow (Tanaka \& Lewin 1995).

For neutron stars, the behavior is strongly influenced by the magnetic field. In sources with strong fields $\left(B \sim 10^{12} \mathrm{G}\right)$ the accreted matter will be swept up by the magnetic field lines several hundred radii from the neutron star and led to the magnetic poles. There, all the energy is released in a very compact region - about $1 \mathrm{~km}^{2}$ - either on the surface of the neutron star or in an extended column above the poles delimited by a shock front in the plasma flow. The radiation spectrum is nonthermal and may include cyclotron line features as direct signature of the magnetic field. If the magnetic poles are not aligned with the rotation axis, pulsations can be observed (White, Nagase \& Parmar 1995).

In sources with weak fields the accretion disk extends close to the neutron star and can even reach its surface. The spectrum from those objects can often be modeled by adding the spectral contribution from the accretion disk - the sum of blackbody spectra at various temperatures - and a contribution from a boundary layer between the disk and the star. Bursts are observed in some of these sources, which can be explained by the sudden ignition of the accreted mass at the surface of the star (Lewin, van Paradijs, Taam, 1995).

At optical wavelengths, emission from the companion star and sometimes from the accretion disk can be observed. Regular variations in the optical lightcurve are often caused by the orbital period of the system. However, in some cases, other phenomena can also be at the origin of the optical variations, such as a precessing warped accretion disk as observed in Her X-1 (Shakura et al 1998). Long-term optical monitoring of Be X-ray binaries has also shown feedback cycles between the neutron star and the mass donor (Negueruela et al. 2001). For black hole systems, a systematic comparison of the erratic variations in the lightcurves at different wavelengths is used to distinguish between different models of the accretion process.

\subsection{Gamma Ray Bursts}

Good spatial position accuracy of GRBs and a fast reaction time after their detection are crucial factors for the multiwavelength analysis of these objects and the determination of their distance.

INTEGRAL aims at a spatial accuracy of 1 arc minute and a reaction time of a few seconds. In order to achieve these goals, the data sent by the satellite is directly forwarded to the ISDC and analyzed in near real time (see Sect. 2). A dedicated software, the INTEGRAL Burst Alert System, automatically searches 
for the occurrence of a GRB, and issues an alert on a positive detection. Internet packets are simultaneously (within a few seconds after the occurrence of the GRB) sent to a pre-registered list of machines around the world giving the position of the GRB. A scientist on duty at the ISDC then analyzes the data to confirm (or possibly invalidate) the alert.

Any interested person can register to the GRB alert system. There are several alert trigger levels, according to the probability at which the alert system estimates the event to be a GRB. Small telescopes around the earth are particularly well suited to respond to the alert and search for an optical counterpart, because they are much more numerous and well-dispersed around the globe than big ones (this increases the probability of having several telescopes which can observe the event under clear night sky) and because they could react quickly if prepared to do so.

\section{Conclusions}

INTEGRAL will continue to nourish a fascinating branch of modern high-energy astrophysics which can greatly benefit from simultaneous multiwavelength observations. World-wide small telescopes may be efficiently involved in those efforts.

Several studies are being conducted by scientists at the ISDC. We have briefly mentioned three of them in Sect. 4. Interested readers involved in small telescopes are welcome to contact us in order to perform optical observations of some of the objects observed by us with INTEGRAL.

\section{References}

Camenzind M. 1997, in Les noyaux actifs de Galaxies, Springer-Verlag.

Lewin W.H.G., van Paradijs J. \& Taam R.E., in Lewin W.H.G., van Paradijs J. \& van den Heuvel E.P.J., eds, X-Ray Binaries, Cambridge Astrophysics Series 26, 1995, pp 175-228

McHardy I., Lawson A., Newsam A., et al. 1999, MNRAS 310, 571

Negueruela I., Okazaki A.T., Fabregat J. et al. 2001, A\&A,369, 117

Paltani S., Courvoisier T.J.-L., Walter R. 1998, A\&A 340, 47

Robson I. 1996, Active Galactic Nuclei, John Wiley \& Sons Ltd.

Shakura N.I., Ketsaris N.A, Prokhorov M. \& Postnov K.A. 1998, MNRAS, 300, 992

Tanaka Y. \& Lewin W.H.G., in Lewin W.H.G., van Paradijs J. \& van den Heuvel E.P.J., eds, X-Ray Binaries, Cambridge Astrophysics Series 26, 1995 , pp 126-168

Türler M., Courvoisier T.J.-L., Paltani S. 2000, A\&A 361, 850

Türler M., Paltani S., Courvoisier T.J.-L., et al. 1999, A\&AS 134, 89

van Paradijs J., et al. 1997, Nature 386, 686

White N.E., Nagase F. \& Parmar A.N., in Lewin W.H.G., van Paradijs J. \& van den Heuvel E.P.J., eds, X-Ray Binaries, Cambridge Astrophysics Series 26,1995 , pp $1-49$ 